\title{
Preliminary Study on a Reduced Scaled Model Regarding the Air Diffusion inside a Crew Quarter on Board of the ISS
}

\author{
Mihnea Sandu ${ }^{1 *}$, Ilinca Nastase ${ }^{l}$, Florin Bode ${ }^{1,2}$, CristianaVerona Croitoru ${ }^{l}$, Laurentiu Tacutu ${ }^{l}$ \\ ${ }^{1}$ CAMBI Research Center, Technical University of Civil Engineering Bucharest,021414Bucharest, Romania \\ ${ }^{2}$ Technical University of Cluj Napoca, Department of Mechanical Engineering 400020 Cluj - Napoca, Romania
}

\begin{abstract}
The paper focus on the air quality inside the Crew Quarters on board of the International Space Station. Several issues to improve were recorded by NASA and ESA and most important of them are the following: noise level reduction, $\mathrm{CO}_{2}$ accumulation reduction and dust accumulation reduction. The study in this paper is centred on a reduced scaled model used to provide simulations related to the air diffusion inside the CQ. It is obvious that a new ventilation system is required to achieve the three issues mentioned above, and the solutions obtained by means of numerical simulation need to be validated by experimental approach. First of all we have built a reduced scaled physical model to simulate the flow pattern inside the CQ and the equipment inside the CQ has been reproduced using a geometrical scale ratio. The flow pattern was considered isothermal and incompressible. The similarity criteria used was the Reynolds number to characterize the flow pattern and the length scale was set at value $1 / 4$. Water has been used inside the model to simulate air. Velocity magnitude vectors have been obtained using PIV measurement techniques.
\end{abstract}

\section{Introduction}

Currently, on the International Space Station (ISS) several personal Crew Quarters (CQ) are installed for the crew members. Functionally a CQ provides an acoustically quiet and visually isolated area in which crew members can sleep, relax, and retreat to a private area (Figure 1).
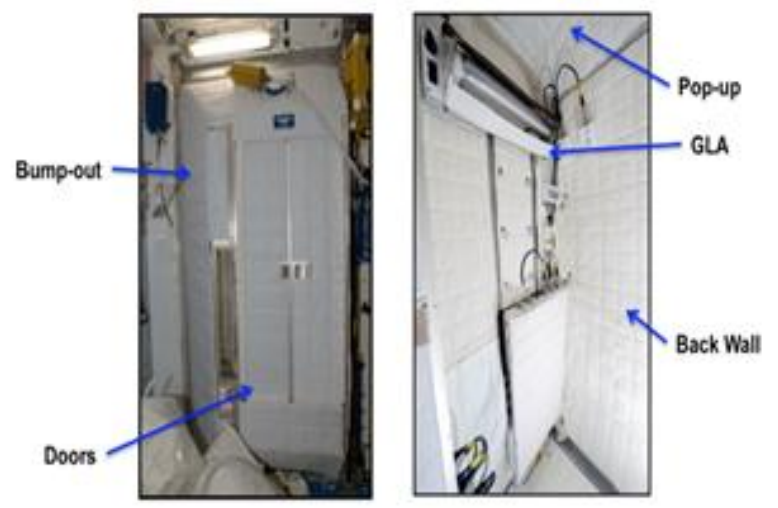

Fig. 1. CQ on-orbit exterior view (left) and interior view (right)

Design and development efforts for long mission crew member's accommodations started with the US Skylab CQ [1]. Skylab featured visual private space for each crew member but lacked of acoustic and light isolation and had very limited ventilation control $[2,3]$. The Russian CQ Kayutas were introduced on Salyut 6 and the same configuration was used on MIR and on the
ISS Service Module. Kayutas CQ provided an increased visually private volume and a $20 \mathrm{~cm}$ diameter window. However the window increased crewmember space radiation exposure. The Kayutas CQ draw air from the module cabin but were generally found to be warm and not to provide enough acoustic attenuation of the cabin noise [3]. The crew uses the CQ primarily for sleeping, but also for performing tasks such as put on or remove clothing, using laptops, private communication, and some minimal personal hygiene.

Each USOS CQ was designed with an individual ventilation system, acoustic mitigation materials, laptop connections, and internet connection to allow crew members personal communication with family and friends [4]. Since their deployment in 2008, the CQ performance has been closely monitored to validate that the design continues to meet requirements. At the beginning, the USOS CQs have received generally favorable crew comments with respect to acoustics [5]. For the control of the airflow, the CQ provides a fan speed switch with three positions and adjustable guide vanes to allow the crew to customize the airflow levels and direction. The crew reported that, in general, the variable fan speed and the guide vanes provided good adjustability although most crew members set the fan speed and the guide vanes to direct air in one direction and leave them in that position. Nominal fan speed varied from crewmember to crew member, but most crew members reported that the fans were kept on medium or high speed formost of the time. Some crew members commented that fans on high speed produced

* Corresponding author: mihnea.sandu@utcb.ro 
too much noise inside the $\mathrm{CQ}$, indicating the need for reducing the acoustic signature of fans at middle and high speed levels of the fans[5]. Most crew members felt that the fan low speed did not provide enough airflow and, due to dust accumulationover time in the CQ ventilation system, the fans at low speed produced a low airflow situation that resulted in caution alarms in CQ.

Finally, the following three issues were reported by NASA and ESA as the most important:

- Noise levels: Since their development [2, 3, 6, 7] engineers deposed a lot of efforts to reduce the acoustic signature of the chosen ventilation solution. In the present concept they had to allocate around $13 \%$ and $6 \%$, respectively of the total mass and volume of a CQ to acoustic reductions. The total impact of the current ventilation solution, in terms of acoustic reduction is respectively $23 \mathrm{~kg}$ and $0.3 \mathrm{~m}^{3}$ for one CQ unit.

- $\mathrm{CO}_{2}$ accumulation issues: Some crew members have been waking up with headaches $[8,9]$. It is believed the CQ has good air flow but there is high $\mathrm{CO}_{2}$ in the station when the Carbon Dioxide Removal Assembly (CDRA) is not functioning to its capacity. Currently NASA is looking for a local solution for scrubbing $\mathrm{CO}_{2}$ from the local air of the CQ to help crew rest better if the CDRA is working poorly [8].

- Dustaccumulation issues: The ISS environment in general and the CQ environment in particular are getting more polluted in terms of particulate matter than expected originally by the engineers [8]. Most of this dust comes from the people themselves. This dust gets into the ducting of the CQ's ventilation system and causes problems with the air flow sensors that are monitoring the flow variation to prevent asphyxiation hazards.

This paper is part of a larger study which implies as main objective the proposal of an optimized concept of the existing CQ on board of the ISS with improved air distribution systems in terms of fans and acoustics. The improved CQ ventilation system will allow the possibilityof a direct adaptation of the existing CQ on the USOS Node 2 module of the ISS to eliminate the current problems of the crew members of the ESA and NASA teams. This will be translated through the improvement of the acoustical performance of the existing CQ on board of the ISS allowing future developments for new concepts of crew accommodations for future long duration and through the increasing of the space dedicated to the crew member in the current CQ design with a reduction of the total mass. The new configuration will also cover the pressure drop related to the introduction of a filter assembly for fine aerosols.

In this respect numerical simulations for the air diffusion system and $\mathrm{CO}_{2}$ diffusion inside the $\mathrm{CQ}$ must be performed and these simulations need experimental validation. This paper deals precisely with the first attempts to create a reduced scaled model for the CQ made by our team. We have focus to model at a smaller scale the air flow pattern and the diffusion of $\mathrm{CO}_{2}$ inside the CQ. We have considered that the velocities and the pressure drops are small enough to consider the fluid incompressible and the temperature is constant so that the main driving force for the $\mathrm{CO}_{2}$ diffusion is the concentration gradient, natural convection being not considered. Another important point is that all experiments will be done in gravitational space which is not present on ISS. Below there is a short description of several similarity criterions and the opportunity to use them for our study.

\section{Similarity Criteria}

Complete similitude between two physical phenomena means that they must be of the same nature and both geometric, dynamic, kinematic similitude and boundary conditions should be accomplished[10]. The geometric similitude requires the proportionality of the corresponding lengths and the equality of angles. The result of the geometric similitude is the length scale. The kinematic similitude requires that if the flow is made of particles, the corresponding particles take the same place inside the flow pattern at same moments in time. As a result, if the kinematic similitude is accomplished between the two phenomena there are a constant length scale and a constant velocity scale. The dynamic similitude implies also the proportionality of the corresponding forces between the phenomena in addition to the geometric and kinematic similitude. The result is obviously a constant force scale.

For this study the dynamic similitude has been found as appropriate. However, the complete similitude between two phenomena of the same nature is impossible to achieve so one must consider only the main forces that govern the flow. It results a restraint dynamic similitude criterion as follow:

- The Froude similitude: the leading forces are the inertia and the gravity forces.

$$
F r=\frac{u}{\sqrt{g L}}
$$

Froude similitude implies the condition $F r_{N}=F r_{M}$. The relation between the scales in case of the Froude similitude is:

$$
\frac{S_{u}^{2}}{S_{g} S_{l}}=1
$$

- The Euler similitude: the leading forces are the inertia and the pressure forces.

$$
E u=\frac{p}{\rho u^{2}}
$$

Euler similitude implies the condition $E u_{N}=E u_{M}$. The relation between the scales in case of the Euler similitude is:

$$
\frac{S_{p}}{S_{\rho} S_{u}^{2}}=1
$$

- The Reynolds similitude: the leading forces are the inertia and the viscosity forces. 


$$
R e=\frac{u l}{\vartheta}
$$

Reynolds similitude implies the condition $R e_{N}=R e_{M}$. The relation between the scales in case of the Reynolds similitude is:

$$
\frac{S_{u} S_{l}}{S_{\vartheta}}=1
$$

- The Peclet similitude: the leading forces are the inertia and the difusivity forces.

$$
P e=\frac{u l}{D}
$$

Peclet similitude implies the condition $P e_{N}=P e_{M}$. The relation between the scales in case of the Peclet similitude is:

$$
\frac{S_{u} S_{l}}{S_{D}}=1
$$

It must be mentioned here that the Peclet number is also found in the literature under the form $P e=\frac{u l}{\alpha}$ where $\alpha$ is called thermal diffusivity and the physical signification in this case is represented by the intensity of the mass convective transfer.

- The Schmidt similitude: the physical meaning of this criteria is represented by the ratio between the intensity of the transfer of the substance by pressure or by concentration differences.

$$
S c=\frac{v}{D}
$$

Schmidt similitude implies the condition $S c_{N}=S c_{M}$. The relation between the scales in case of the Schmidt similitude is:

$$
\frac{S_{v}}{S_{D}}=1
$$

It is obvious that $\boldsymbol{P e}=\boldsymbol{R} \boldsymbol{e} \boldsymbol{S}$.

\section{Experimental Setup Conception}

The next step is to establish the similarity criteria used. Considering the nature of the phenomena and the fact that not all the criteria of similarity can be satisfied simultaneously, we considered that the determinant for the studied phenomenon is the Reynolds criterion defining the flow structure with major implications in the heat transfer as well as the Peclet criterion with major implications in the diffusion mass transfer. First, the interest was tosimulate the $\mathrm{CO}_{2}$ diffusion in air. Here we have identified two issues: the first issue is to find a way to simulate the microgravity, more precisely the fact that there is a difference between the $\mathrm{CO}_{2}$ density and the air density $\left(\mathrm{CO}_{2}\right.$ is heavier) which is very important in gravitational field but not in microgravity space. This issue can be solved by using two fluids with the same density to simulate the diffusion of $\mathrm{CO}_{2}$ in the air in microgravity conditions. In this case the effects of gravity are canceled by the buoyancy forces. If the temperature is kept constant inside the fluid body, natural convection is also avoided. Thus, the only sources of motion between two liquids are forced convection, molecular diffusion and, in some cases, gradients of surface tension. Research on fluid behavior in space is oriented towards the behavior of liquids in the absence of gravity. On Earth, gravitational effects often cancel other influences, but in space, in an essentially no-gravitational environment, the importance of other factors increases. Such a factor is the gradient of surface tension that exists between two different liquids. This gradient can be caused by differences in concentration or temperature and, in both cases, causes mass transfer between two fluids (the Marangoni effect).

Since the experimental scale model will introduce a liquid jet into an existing body of water, a question arises as to the influence of other factors on the liquid behavior. The bibliographic studies on the movement induced by the surface tension gradient in the neardensity systems lead to the conclusion that these effects are not relevant to our case.

Therefore, we have tried a low-scale model in which $\mathrm{CO}_{2}$ airborne diffusion in air was modeled by means of Rhodamine diffusion in water. The choice of rhodamine diffusion in water was imposed by the possibility to use laser induced fluorescence techniques to measure level of concentration. In this respect, using the Peclet criterion and considering that $\mathrm{S}_{\mathrm{D}}$ in this case is equal to about $3,38 \times 10^{-5}$ for a scale of $S_{L}=1 / 4$ lengths, the scale of the $S_{\mathrm{v}}$ should be about $1,35 \times 10^{-4}$. It means that the velocity values on the reduced model should be about $10^{4}$ times smaller than the real values which was not possible to measure with common PIV techniques.

We drop off for the moment the idea of modelling the $\mathrm{CO}_{2}$ diffusion and we have tried to find a way to simulate the flow pattern inside the CQ using Reynolds criterion. Water was kept as working fluid because of technical reasons. In this case considering that the viscosity scale is $S_{v}=1 / 15$ and using $S_{L}=1 / 4$ for the length it results a $4 / 15$ scale for the velocity.

The plans and the main dimensions of the reduced scaled model are showed in figure 2
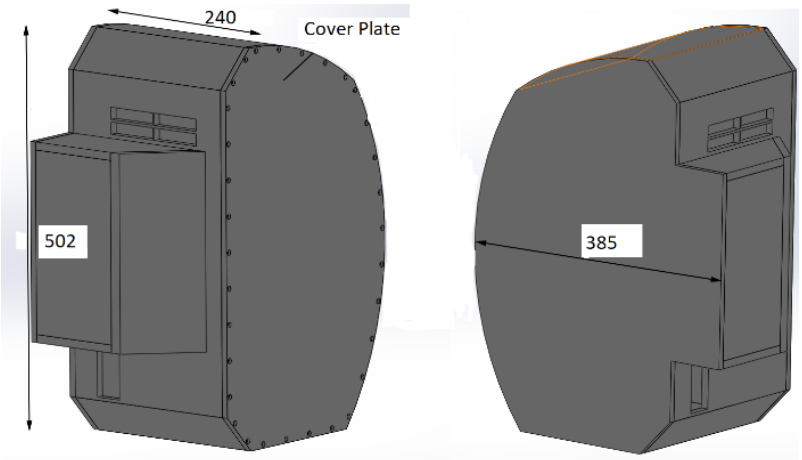

Fig. 2. Main dimensions and shape of the reduced scale model 
The model was made of polycarbonate plates with a suitable refraction index to allow velocity measurement using PIV techniquesas shown in figure 3a,b,c:
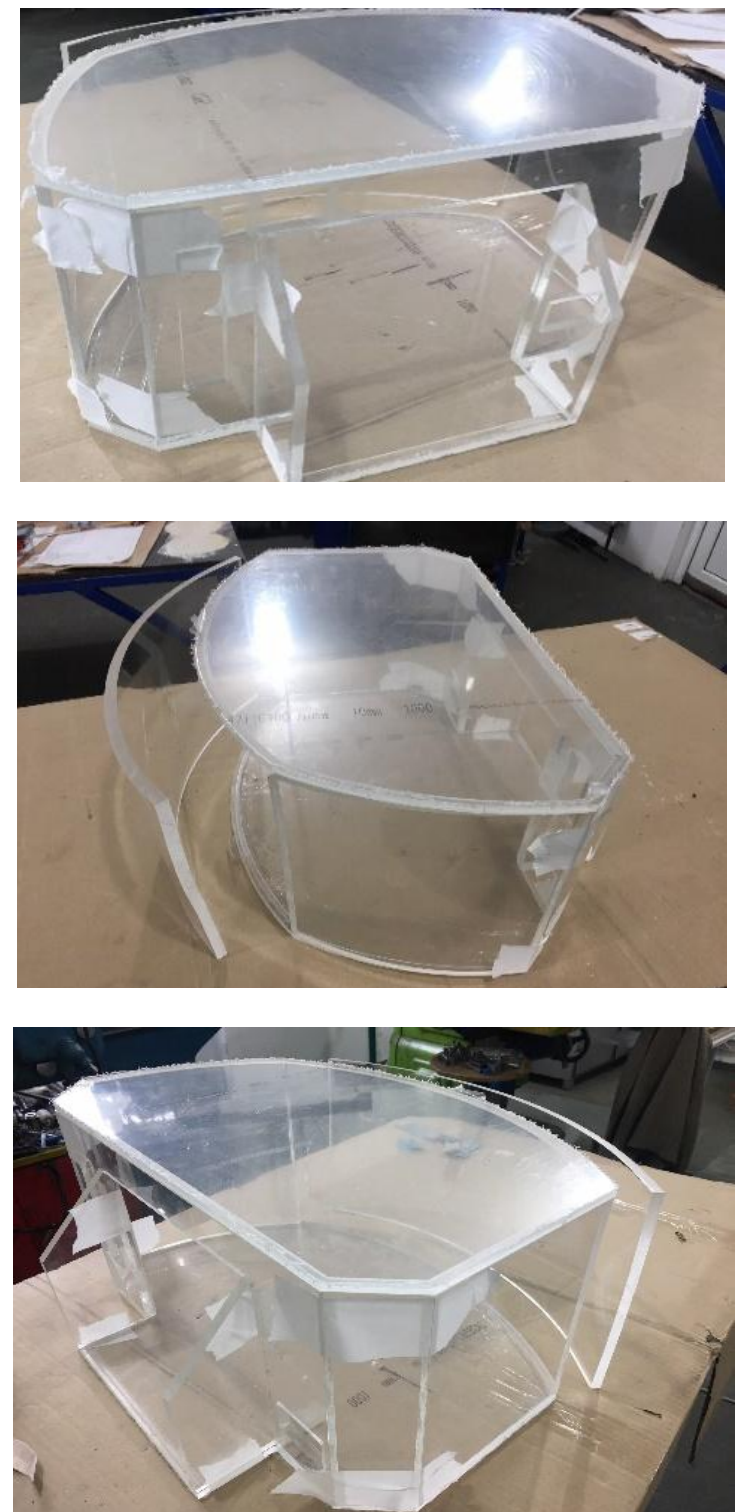

Fig. 3.a,b,cReduced Model for the CQ.

The model was integrated in a closed water loop using a small pump for ensuring the water flow rate. The main purpose was to prove the feasibility of the model, precisely to demonstrate how to determine the velocity field inside the reduced scaled model.

In this study, we have employed a Dantec Particle Image Velocimetry (PIV)system for the velocity field measurements. This system is composed of one high sensitivity Flow Sense $4 \mathrm{M}$ camera of $4 \times 10^{6}$ pixels resolution and of a Dual Power $200 \mathrm{~mJ}$ laser having the wave length of $532 \mathrm{~nm}$. The acquisition frequency of the PIV system was $7.5 \mathrm{~Hz}$. The water flow was seeded with hollow glass spheres with a mean diameter of $50 \mu \mathrm{m}$. The images calibration gave a spatial resolution of $100 \mu \mathrm{m}$ per pixel which is corresponding to a $200 \times 200 \mathrm{~mm}^{2}$ field of view.
The laser has been placed above the model and the camera from a lateral side of the model as in the figure 4 below.
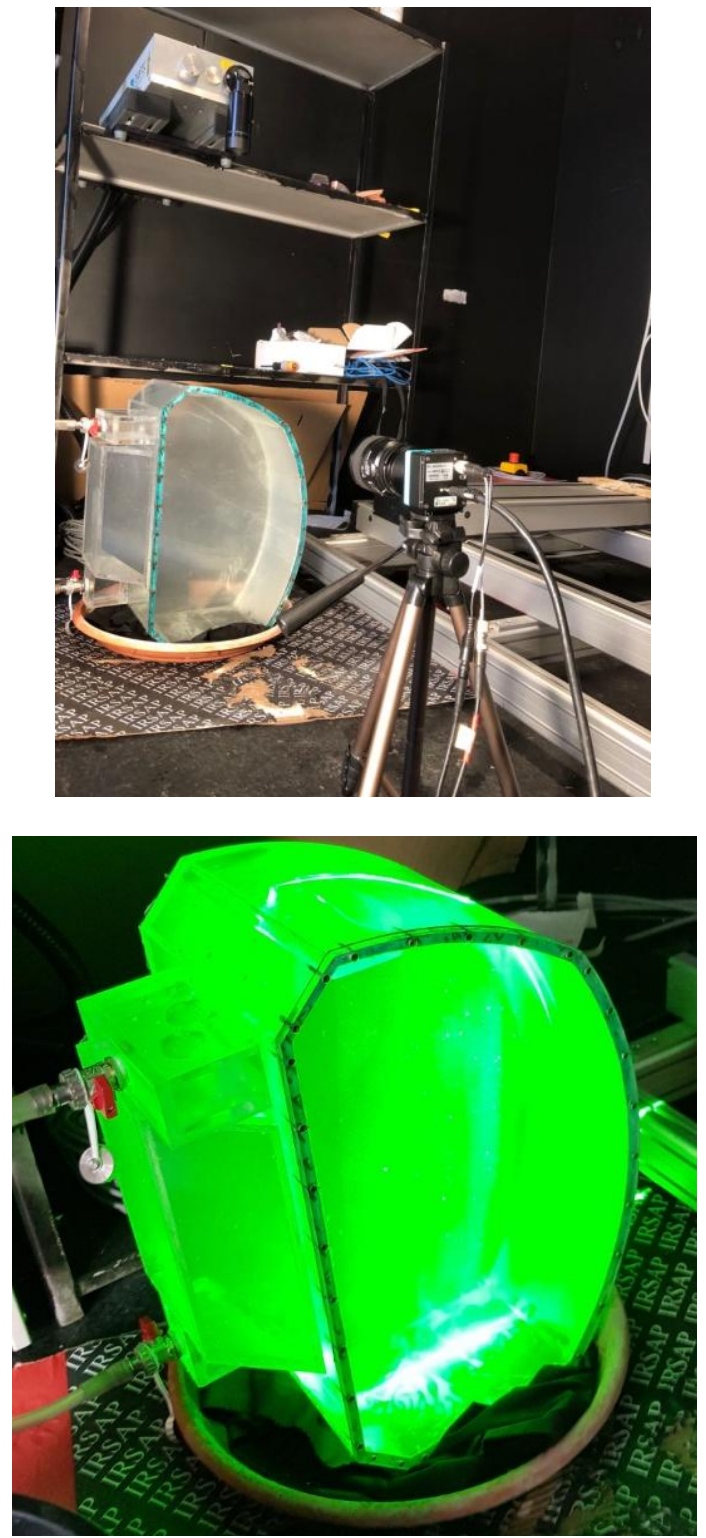

Fig. 4.a,bExperimental setup for PIV measurements

\section{Results}

The velocity field has been measured in a plane parallel to the lateral face of the model as indicated in figure 5 . Several other planes and interesting configurations should be investigated but given the fact that the main purpose was to prove the feasibility of the model all these experiments will be accomplished following the future numerical simulations that will be part of this study. 


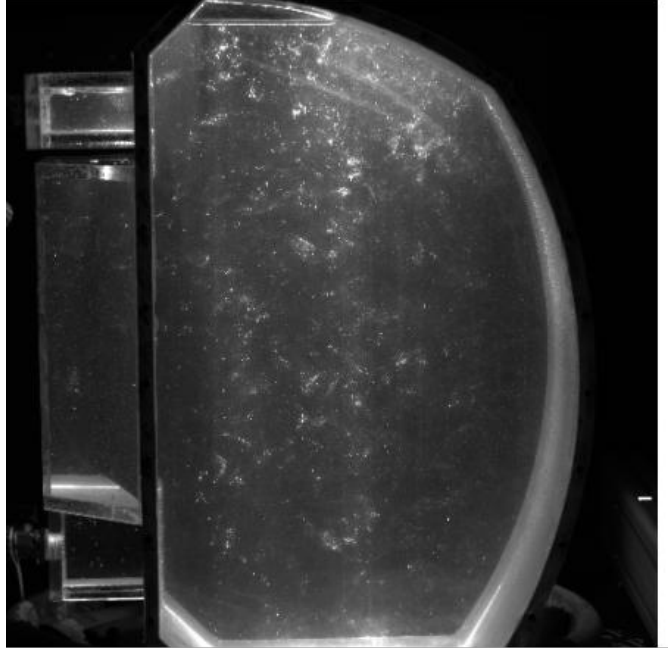

Fig. 5. Flow visualisation in plane parallel to the lateral face of the model

The velocity vectors measured are shown on the figure 6 below. The measured velocity magnitudes are less the $0.05 \mathrm{~m} / \mathrm{s}$.

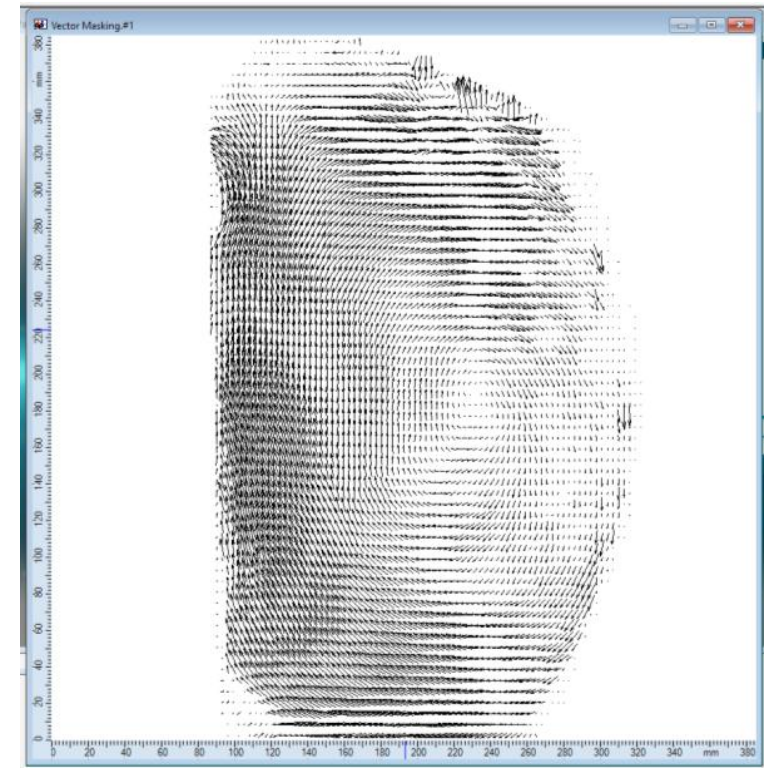

Fig. 6. Velocity vectors

\section{Conclusions and perspectives}

Even if the study is a preliminary one, some conclusions and some interesting perspectives can be outlined of it. It is obvious from the beginning that all the experiments have been done in gravitational field which is not the case in space. This is the main reason to use two substances with nearly the same density for modelling the $\mathrm{CO}_{2}$ diffusion in air but due to our limited measurement possibilities we had no results. However, we have continued using water inside the model to simulate the flow pattern with respect to Reynolds criterion. This preliminary study guide us towards the following conclusions:

- The $\mathrm{CO}_{2}$ diffusion in air should be modelled on a reduced scaled model using the Peclet criterion. Two gases with nearly the same density are needed in order to avoid the gravity effects. Using water as the main fluid is not appropriate because the diffusivity coefficients values for different substances (tracers) in water are $10^{5}$ times smaller than the $\mathrm{CO}_{2}$ diffusion coefficient in air and the corresponding scales for length and velocity are not technically feasible.

- The results have shown that it is possible to use water on the model to simulate the flow pattern with respect to Reynolds criterion. The use of the water is indicated for this case because it is easier to measure using PIVtechnique and the insemination with particles is better than for gases.

- The similitude regarding Peclet and Reynolds criterion cannot be achieved at the same time, the physical phenomena must be splitted.

- A reduced scaled model using gases as working fluids need to be tested also with respect to the Reynolds criterion. The advantage here is that the microgravity could be simulated better for this case.

This work was supported by a grant of the Romanian space agency ROSA, QUEST - Advanced air diffusion system of the crew quarters for the ISS and deep space habitation systems, STAR-CDI-C3-2016-577

\section{References}

1. P.O.Wieland, Living Together in Space: The Design and Operation of the Life Support Systems on the International Space Station NASA/TM-1998206956/1. (1998).

2. J.L.Broyan, M.A. Borrego, and J.F. Bahr. International Space Station USOS Crew Quarters Development. in 08ICES-0222. (2008).

3. C.Adams, Four Legs in the Morning: Issues in CrewQuarter Design for Long-Duration Space Facilities. in 28th International Conference on Environmental Systems, Danvers, MA. (1998).

4. NASA Reference guide to the International SpaceStationhttps://www.nasa.gov/pdf/508318main ISS_ref_guide_nov2010.pdf. (2010).

5. T.P.Schlesinger, B.R. Rodriguez, and M.A. Borrego, International Space Station Crew Quarters On-Orbit Performance and Sustaining Activities. AIAA Journal, (2013).

6. J.L.Broyan, S.M. Cady, and D.A. Welsh, International Space station Crew Quarters Ventilation and Acoustic Design Implementation. AIAA Journal, (2010).

7. S.Fairburn, and S. Walker, 'Sleeping With the Stars'-The Design of a Personal Crew Quarter for 
the International Space Station. 2001, SAE Technical Paper.

8. 2015-2016 HUNCH Design \& Prototyping program https://www.nasahunch.com/design-prototype/. (2015).

9. J.James, The Headache of Carbon Dioxide Exposures in SAE Technical Paper 2007-01-3218, 2007, doi:10.4271/2007-01-3218. (2007).

10. M.Degeratu, Analiza dimensionala, similitudine si modelare. (2015), Ed. Academiei Oamenilor de Stiinta Romania. 